\title{
Time domain astronomy from Dome C: results from ASTEP
}

\author{
J.-P. Rivet ${ }^{1}$, L. Abe ${ }^{1}$, K. Agabi ${ }^{1}$, M. Barbieri ${ }^{1}$, N. Crouzet $^{2}$, \\ I. Goncalves ${ }^{1}$, T. Guillot ${ }^{1}$, D. Mekarnia ${ }^{1}$, J. Szulagyi ${ }^{1}$, J.-B. Daban ${ }^{1}$, \\ C. Gouvret ${ }^{1}$, Y. Fantei-Caujolle ${ }^{1}$, F.-X. Schmider ${ }^{1}$, T. Furth ${ }^{3}$, \\ A. Erikson ${ }^{3}$, H. Rauer ${ }^{3}$, F. Fressin ${ }^{4}$, A. Alapini ${ }^{5}$, F. Pont $^{5}$ \\ and S. Aigrain ${ }^{6}$ \\ ${ }^{1}$ Laboratoire Lagrange, UMR7293, Université de Nice Sophia-Antipolis, CNRS, Observatoire \\ de la Côte d'Azur, BP 4229, F-06304 Nice Cedex 4 France. \\ ${ }^{2}$ Space Telescope Science Institute, Baltimore, MD 21218, USA \\ ${ }^{3}$ DLR Institute for Planetary Research, 12489 Berlin, Germany \\ ${ }^{4}$ Harvard-Smithsonian Center for Astrophysics, Cambridge, MA 02138, USA \\ ${ }^{5}$ School of Physics, University of Exeter, Stocker Road, Exeter EX4 4QL, United-Kingdom \\ ${ }^{6}$ Department of Physics, University of Oxford, Oxford OX1 3RH, United Kingdom
}

\begin{abstract}
ASTEP (Antarctic Search for Transiting Exo Planets) is a research program funded mainly by French ANR grants and by the French Polar Institute (IPEV), dedicated to the photometric study of exoplanetary transits from Antarctica.

The preliminary "pathfinder" instrument ASTEP-South is described in another communication (Crouzet et al., these proceedings), and we focus in this presentation on the main instrument of the ASTEP program : "ASTEP-400", a $40 \mathrm{~cm}$ robotized and thermally-controlled photometric telescope operated from the French-Italian Concordia station (Dome C, Antarctica).

ASTEP-400 has been installed at Concordia during the 2009-2010 summer campaign. Since, the telescope has been operated in nominal conditions during 2010 and 2011 winters, and the 2012 winterover is presently in progress. Data from the first two winter campaigns are available and processed. We give a description of the ASTEP-400 telescope from the mechanical, optical and thermal point of view. Control and software issues are also addressed. We end with a discussion of some astronomical results obtained with ASTEP-400.
\end{abstract}

Keywords. Transiting exoplanets, photometry, Dome C

\section{Introduction}

Since the first discovery, in 1999, of an exoplanet (HD209458b) transiting across its host star (Charbonneau et al. 2000, Henry et al. 1999), more than 282 transiting exoplanets have been discovered around 230 stars. The transit method has revealed to be a powerful method to detect new exoplanets, and also to characterize already known ones, as a complement to the radial velocity method.

Several ground based instruments are at least partially dedicated to transiting exoplanet studies. For example, HATNet is a network of 6 robotic $11 \mathrm{~cm}$ wide field telescopes spread around the word, dedicated to variable stars and transiting exoplanets search (Bakos et al. 2007). It has discovered so far 41 transiting exoplanets. The WASP project (Christian et al. 2006) has discovered 79 with a pair of wide angle small photometric telescopes (SuperWASP I and II) located in La Palma and at Sutherland in South Africa respectively. Other ground-based transiting exoplanets surveys (MEarth project, OGLE, BEST, ...) have also contributed to the 282 discoveries. More recently, space 
borne instruments have joined the race (CoRoT: Baglin et al. 2006; Kepler: Borucki et al. 2010), yielding an important increase in the exoplanet detection rate and in photometric accuracy.

The periods of the 282 known exoplanets range from 0.45 to more than 300 days. Thus, long time series of photometric data are required for exoplanet discovery and qualification. Moreover, the photometric drop during the transit is very low. Even for a Jupiter-like planet orbiting a solar type star, the depth of the drop in the lightcurve hardly reaches $1 \%$, and much lower depths are frequent. Thus high accuracy photometric data are required, with sub-millimagnitude noise (white and red). Consequently, observations from a good photometric site in polar regions would be of interest to discover new exoplanet candidates, but also to perform follow-up observations of known systems, for a better characterization.

The Dome C site (Antarctic Plateau; $75^{\circ} 06^{\prime}$ South, $123^{\circ} 19^{\prime}$ East, altitude: $3250 \mathrm{~m}$ ) has been thoroughly tested as far as atmospheric turbulence parameters (seeing, isoplanatic angle and outer scale) are concerned (Agabi et al. 2006; Ziad et al. 2008; Aristidi et al. 2009). However, little was known about the photometric quality of the site.

The aim of the ASTEP program is twofold : first, assessing the photometric capabilities of Dome C, and second, studying transiting exoplanets (discovery and follow-up). ASTEP is a two-instrument program: ASTEP-South and ASTEP-400, both installed at the French-Italian Concordia station (Dome C).

ASTEP-South is a small "pathfinder" instrument observing the South pole region since 2008. It is a commercial $10 \mathrm{~cm}$ refractor on a commercial mount. No motor is needed, since the target is the south celestial pole. The field of view covered by the $4096 \times 4096$ pixels camera is $3.88^{\circ} \times 3.88^{\circ}$. The data from the first season of observation (2008) has revealed the good photometric quality of the Dome $\mathrm{C}$ site (Crouzet et al. 2010). Results from the 2008 to 2011 seasons are discussed at length in the presentation by N. Crouzet in this symposium (Crouzet et al. 2012), and thus, fall outside of the scope of the present contribution.

ASTEP-400 is a $400 \mathrm{~mm}$ semi-robotic custom telescope on a commercial equatorial mount, in operation at the Concordia station since 2010. The instrument itself and the results from its first winter observation campaign are described here.

This paper is organized as follows. We first recall basic facts about the ASTEP program, and give a detailed description of the ASTEP-400 instrument, data, and reduction pipelines. Then, we discuss the results of the 2010 and 2011 winter campaigns (exoplanets candidates found, follow-up of WASP-19b transit). We end up with some conclusions about the photometric accuracy of ASTEP-400.

\section{The ASTEP program}

\subsection{The context}

The ASTEP program (Antarctic Search for Transiting Exo-Planets) has been proposed in 2005 by F. Fressin, during his PhD at the Observatoire de la Cote d'Azur (France). The funding, by an ANR grant, was obtained in 2006, and the "pathfinder" instrument ASTEP-South was installed at the Concordia station in late 2007, ready for operation in the 2008 winter campaign (1,500 hours of data delivered in 2008).

The main instrument, ASTEP-400, was tested in France from March to October 2009, and installed at Concordia in December 2009, and ready for operation at the beginning of the 2010 austral winter (daytime first light late December 2009; nighttime first light on 


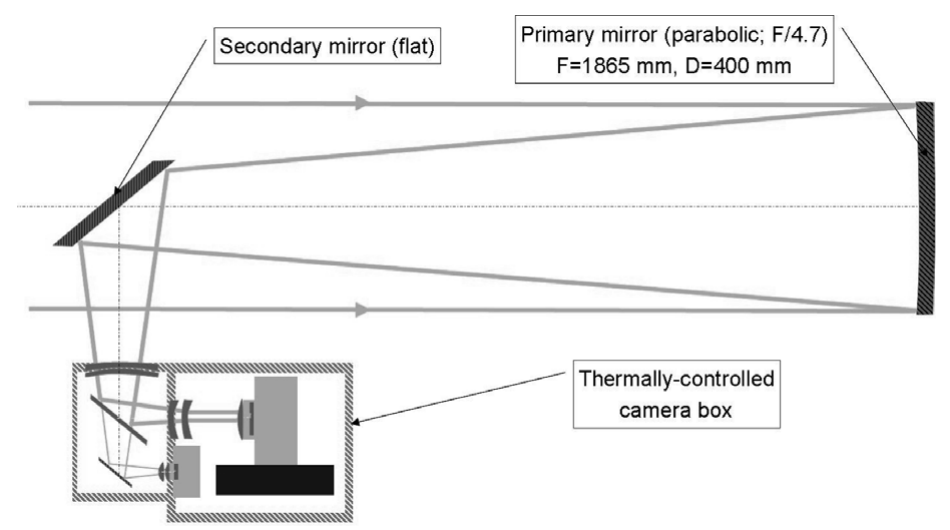

Figure 1. ASTEP-400 optical scheme.

March $25^{t h}, 2010$ ). Presently (September 2012), the third winter season of ASTEP-400 and the fifth for ASTEP-South is about to end.

The ASTEP collaboration was initiated by the University of Nice-Sophia-Antipolis and the Observatoire de la Cote d'Azur (France). It includes the Deutschen Zentrum für Luft- und Raumfahrt (Germany), the Exeter University (UK), the Observatoire de Haute Provence and the Observatoire de Marseille (France).

\subsection{The ASTEP-400 instrument}

ASTEP-400 is a custom $400 \mathrm{~mm}$ Newtonian telescope with coma corrector, designed to withstand both summer and winter temperatures at Dome $\mathrm{C}$, that is, temperatures ranging from $-20^{\circ} \mathrm{C}$ to $-75^{\circ} \mathrm{C}\left(-4^{\circ} \mathrm{F}\right.$ to $\left.-103^{\circ} \mathrm{F}\right)$, with rapid variations. Figure 1 sketches the ray path of the optical design.

The custom five-lens comma corrector yields a $1^{\circ} \times 1^{\circ}$ field of view on a $4096 \times 4096$ pixels FLI Proline camera. The plate scale is $0.88^{\prime \prime}$ per pixel $(1$ pixel $=9 \mu \mathrm{m})$. The PSF (Point Spread Function) is stable and homogeneous from the centre to the corner of the CCD, with a full width at half-maximum (FWHM) below 3 pixels when atmospheric turbulence is low (the entrance pupil is at less than $2 \mathrm{~m}$ above the ice surface, which is far from optimal for the seeing conditions).

To achieve a good thermal and mechanical stability, the structure of the telescope is an 8-th order double Serrurier assembly with carbon fiber legs, connected to an aluminium alloy main frame through Invar sleeves. To protect the optics both against stray light and ice dust, the Serrurier structure is covered by a two-layer cloth envelope.

Even with this careful design and manufacturing, the thermal expansion during rapid temperature variations may yield minor but unacceptable defocus $(0.15 \mathrm{~mm}$ for a temperature variation of $30^{\circ} \mathrm{C}$ according to a finite elements simulation with NASTRAN). Thus, the science camera stands on a motorized, computer-controlled translation stage.

For accurate guiding, the incoming beam is split by a dichroic plate, which reflects the light with $\lambda>600 \mathrm{~nm}$ to the science camera, and transmits the light with $\lambda<600 \mathrm{~nm}$ to an auxiliary guiding camera, a SBIG ST402. In normal observing conditions, the resulting guiding accuracy is about $0.5^{\prime \prime}$ or better.

The science and guiding cameras, the motorized translation stage, and the electronic interfaces for the camera signals cannot withstand the Antarctic temperatures. Thus, a multi-zone thermally controlled casing has been designed to maintain the cameras and their surroundings at temperatures compatible with safe operations. The thermal control is done by electric heating resistors, PT100 temperature sensors and commer- 


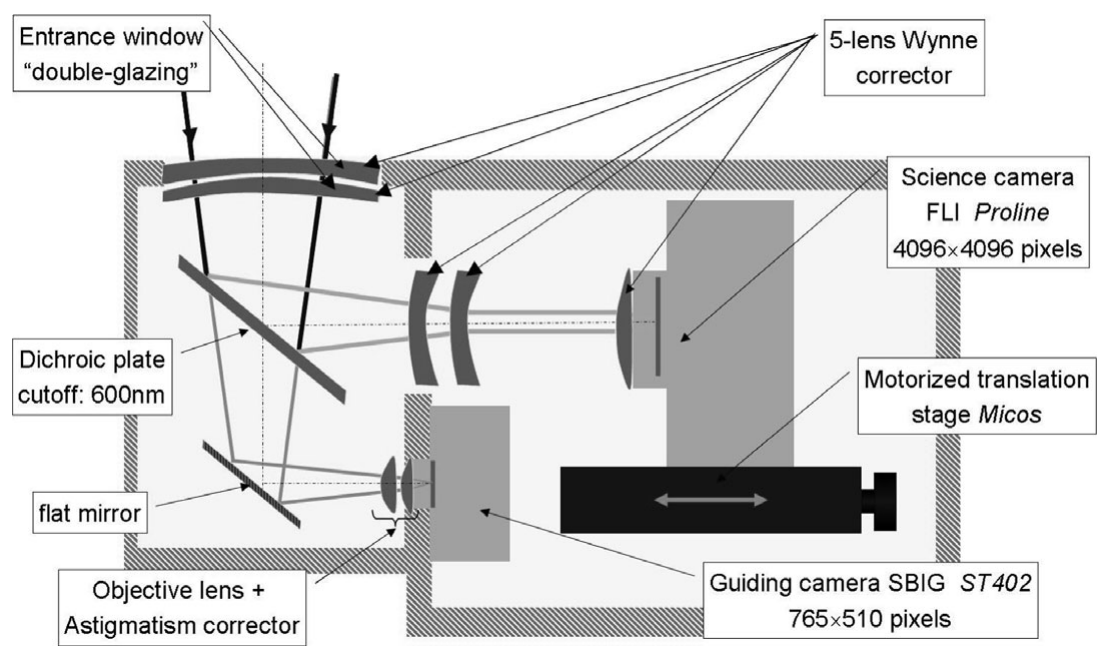

Figure 2. A simplified sketch of the thermally-controlled camera box.

cial computer-supervised controllers. This "camera box" (see Figure 2) receives the light from the Newtonian telescope's flat secondary mirror through the two first lenses of the 5-lens Wynne coma corrector, which act as a "double glazing" insulation window. The first compartment, which contains only optical components (dichroic plate, M3 mirror) is stabilized around $-20^{\circ} \mathrm{C}\left(-4^{\circ} \mathrm{F}\right)$. The second compartment, which contains the cameras and electronic devices, is held around $-10^{\circ} \mathrm{C}\left(+14^{\circ} \mathrm{F}\right)$, and the cameras' front panel is held at $+5^{\circ} \mathrm{C}\left(+41^{\circ} \mathrm{F}\right)$, since the mechanical shutters cannot withstand too low temperatures. All the thermal controllers are daisy-chained on a RS485 bus, which communicates with the main control computer through a RS485/RS232 adapter. So, all temperatures can be monitored, and the set points of the controllers can be adjusted by software.

In Antarctic winter conditions, frost can form and/or ice dust can fall on the primary mirror of the telescope, and, to a lesser extent, on the secondary mirror. During the first winter season (2010), this problem required direct human intervention to manually defrost the mirror, or to remove the ice dust with a soft brush. To reduce direct human intervention as much as possible, we have introduced a defrosting mechanism for the primary and secondary mirrors during the 2010-2011 summer campaign. This system consists of custom designed film resistors, attached to the rear surfaces of the primary and secondary mirrors. Temperature probes are glued on the side of these mirrors, and a pair of additional commercial temperature controllers are added to drive the heaters. Since these controllers are also daisy-chained with all other controllers, they communicate with the mainframe computer driving the telescope. So, the mirror temperatures can be monitored easily, and the power supplied to the heaters can be adjusted by the winter crew, from the control room.

This defrosting system can operate in two different modes : a "preventive" mode and a "curative" mode. In the preventive mode, a small percentage of the maximum power is supplied to the heaters, so as to maintain the mirror surface a few degrees above ambient temperature (less than $4^{\circ}$ for the primary, and less than $2^{\circ}$ for the secondary). This mode can run during observations without hampering the image quality. Figure 3 shows typical temperature curves of the primary and secondary mirrors, for the preventive mode. Under special wether conditions frost can appear, or, more frequently, ice dust grains can fall on the optics. Then, the curative mode is activated, and $100 \%$ of the nominal power of the heaters is supplied. This removes the frost or ice dust. Of course, no image can be 


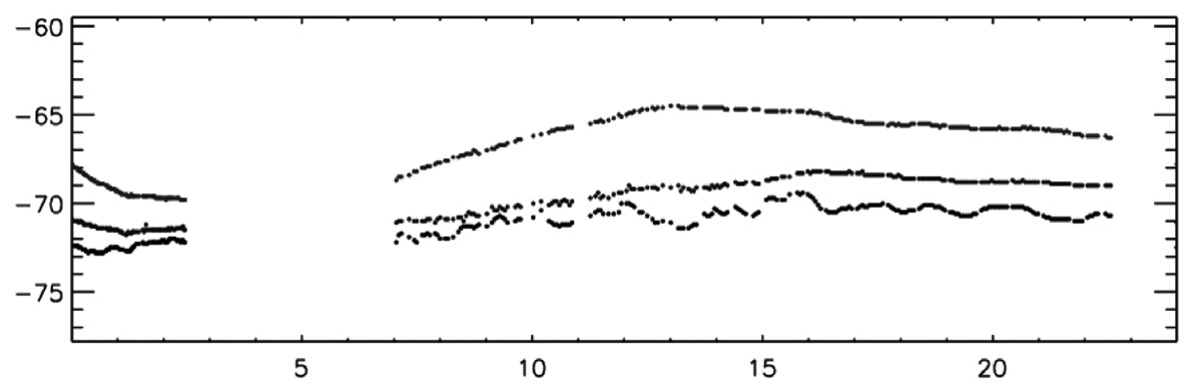

Figure 3. Temperatures (Celsius) of the primary mirror (upper curve), of the secondary mirror (middle curve) and outside temperature (lower curve), as a function of the UT time, on July $17^{\text {th }}, 2012$.

taken during this curative phase. This system was used during the 2011 and 2012 winter campaigns.

The telescope is semi-robotic and fully computer-controlled, so that little intervention is required from the winter-over crew. Only regular inspection of the primary mirror may be needed. Through a single graphic user interface, the winter crew member in charge with ASTEP-400 can monitor the relevant parameters of the telescope, perform any modifications, send orders to the mount motors, or run pre-determined observation scripts.

\subsection{The data and the reduction strategy}

Each frame produced by th FLI Proline camera is 32 MiB. A typical observation "night" produces some 400 frames, including calibration frames. This corresponds to more than $12 \mathrm{GiB}$ of data. The 2010 winter season produced around 6 TiB. Since the internet connection at Concordia is not sufficient for bulky data transfer, all these data are transferred to France on hard disks in one of the summer crew members' personal luggage, at the end of the following summer campaign. Thus, data from the winter campaign number $n$ are available in France for processing on February of year $n+1$, typically.

Four types of frames are produced by the science camera: science frames, bias (offset) frames, dark frames, and "sky-flat" frames. Indeed, it is not possible to do either twilight flats or dome flats. So, the photometric gain calibration is performed with "sky-flat" frames. To get these frames, we take 200 frames during the few hours where the sky background rises as the Sun comes close to the horizon. Between each of these frames, a slight random shift is applied, to both the declination and right ascension axes of the telescope. Star images, which are visible on individual frames, are eliminated by taking the median of all these individual frames.

The ASTEP team uses two distinct data processing pipelines: a fast preliminary pipeline running daily at the Concordia station, and a more thorough one, running in France, on more powerful computers, when the data are available (February of Year $n+1$ for the winter season of Year $n$ ).

The fast pipeline is a custom IDL code making extensive use of "Daophot" routines. It first performs standard frame calibrations (bias, dark, flat). Then, it extracts the photometry of all stars in each frame by aperture photometry, to obtain raw light curves for all the stars brighter than the $16^{\text {th }}$ magnitude (in $\mathrm{R}$ band). This yields light curves for several thousands of stars in a typical field. To reject transparency variations, a global calibration is performed on these light curves : the pipeline selects 4,8 or 16 "reference" stars with stable fluxes, and corrects the light curves of other stars accordingly. The reference stars are chosen so as to minimize the resulting photometric noise on the whole 


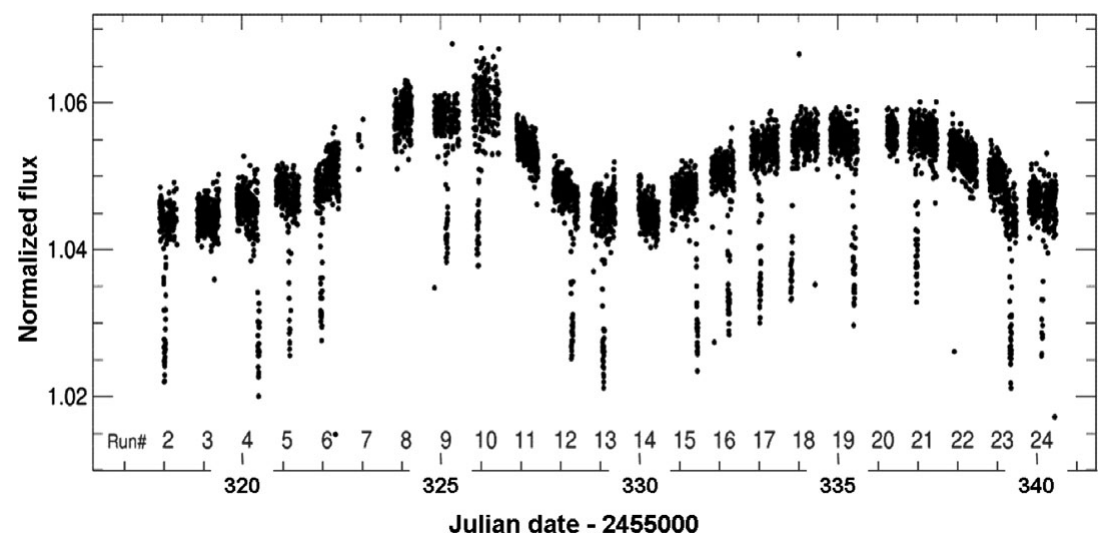

Figure 4. WASP19 lightcurve from May $1^{\text {st }}$ to May $23^{r d}$, 2010. The 10.5 day stellar variation has not been removed.

field. Finally, the BLS transit detection algorithm (Box Least Square, Kovacs et al. 2002) is applied to the calibrated light curves, to extract the lightcurves with interesting features (exoplanets transits, eclipsing binaries, variable stars).

A more thorough custom pipeline has been written, to obtain more refined lightcurves. Being much more time-consuming, this IDL pipeline cannot run on the computers at Concordia station, but only in France. In addition to the standard frame calibrations, this pipeline also corrects for the so-called "sky concentration" effect (Andersen et al. 1995; Bellini et al. 2009). Then, the OIS method (Optimal Image Subtraction, Alard \& Lupton 1998, Miller et al. 2008) is applied, and the raw light curves for all the measurable stars in the field are extracted. These raw lightcurves are calibrated against reference stars, chosen to minimize the residual photometric noise, as for the fast pipeline.

On lightcurves corresponding to exoplanet transit candidates, stellar variation is removed to flatten the lightcurve. Then, the lightcurve is phase-folded and the primary and secondary transits are fitted with the analytic transit model by Mandel \& Agol (2002) with the Levenberg-Marquardt algorithm, to obtain the transit parameters.

\section{Results from the 2010 winter campaign}

\subsection{Photometric accuracy}

To assess the photometric quality of the lightcurves from ASTEP-400, we have studied a well-known transiting exoplanet: WASP-19b (Hebb et al. 2010) This exoplanet transits across its host star $(M v=12.3)$ with a very short period : 0.78884 day. Each individual frame had an exposure time of $130 \mathrm{~s}$. The lightcurve extends from May $1^{\text {st }}$ to May $23^{\text {rd }}$, 2010 (see Figure 4).

The stellar variability is estimated through a median filtering of the original lightcurve, and the resulting filtered contribution (period 10.5 \pm 0.2 days, consistent with Hebb et al. 2010) is subtracted out. This leads to a flattened light curve, which is phase-folded, binned over 0.001 phase units, and fitted with the analytic transit model of Mandel \& Agol (2002). Figure 5 shows the folded lightcurve (binned and un-binned), and the best fitting transit curve for primary and secondary transit.

The rms photometric accuracy is estimated on out-of-transit data. This leads to 3 millimagnitudes for the un-binned data, and to 0.5 millimagnitudes or less, for binned data. This is comparable to using telescopes 2 times bigger at other good temperate astronomical sites. 


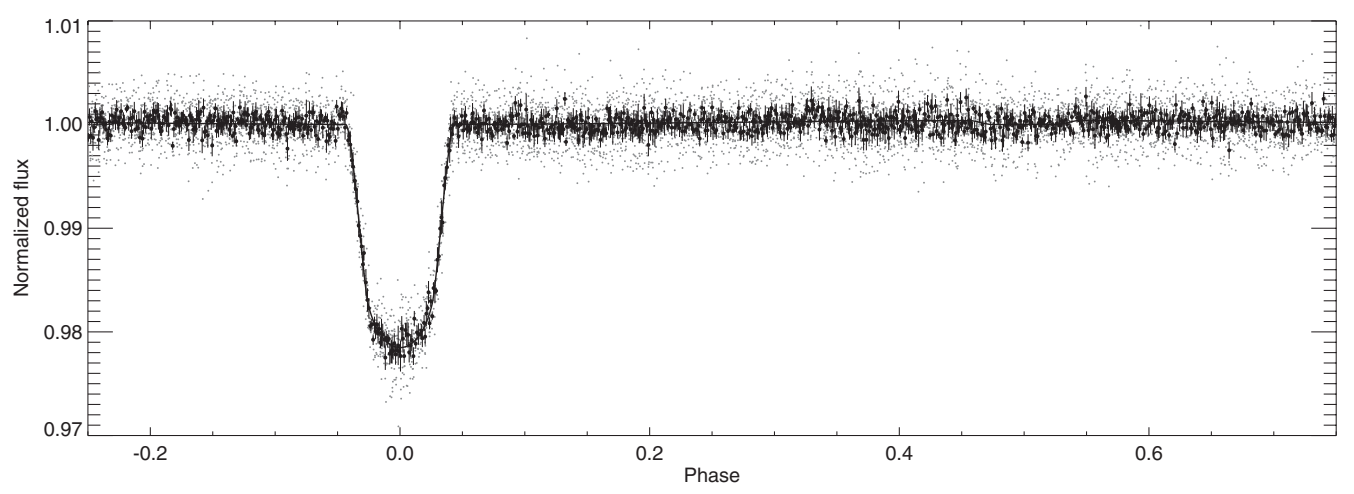

Figure 5. WASP19 lightcurve from May $1^{\text {st }}$ to May $23^{\text {rd }}$, 2010. The stellar variation has been removed and the curve is phase-folded. The small grey dots correspond to individual frames. The large black dots with error bars correspond to data binned over 0.001 phase unit, and the solid curve is the best fit of the primary and secondary transit by the analytic transit model of Mandel \& Agol (2002).

To estimate the contribution of red noise for the 1 hour time scale (typical for transit durations), we have applied the prescription in Pont et al. (2006) on each of the 23 "nights" during which WASP-19 has been observed with ASTEP-400. This yields the following statistics : the median value of the 1 hour time scale red noise is 0.7 millimag. The best and worst values are respectively 0.3 millimag and 2 millimag. In this sample of 23 "nights", one third are less than ideal, with red noise around 1 millimag; some nights are very good, with red noise below 0.4 millimag, and most nights are "average", with red noise around 0.7 millimag. These statistics are typical of very good ground-based photometric sites.

\subsection{Exoplanet candidates}

Only the data from 2010 and 2011 winter seasons have been processed so far. In these data, the BLS algorithm has found 93 lightcurves displaying transit-like structures. Among those, 8 targets are potential exoplanet candidates, with transit depths below $3 \%$ and periods ranging from 0.68 to 4.4 days. Follow-up observations are in progress, to obtain the stellar type of the host star, and to estimate the radial velocity.

\section{Conclusion}

ASTEP is a two-instrument program dedicated to exoplanet discovery and follow-up from Dome C (Antarctic Plateau), and to site qualification for long time scale high duty cycle photometry.

ASTEP-South, a small "pathfinder" instrument, has been in operation at Dome C since 2008, and its fifth winter season is currently in progress.

ASTEP-400, a $400 \mathrm{~mm}$ custom Newtonian telescope on a commercial mount, was installed at Dome C in less than 2 months during the 2009-2010 summer campaign. This instrument has been in operation since the 2010 austral winter, and is presently in its third winter season.

The data from the first two winter seasons have led to lightcurves with white noise around 3 millimag r.m.s. for un-binned data, and less than 0.5 millimag r.m.s. for binned data on a $M_{v}=12.3$ star with 130 seconds exposures. This is comparable to a telescope twice as big in a good temperate astronomical site. 
The red noise statistics (worst: 2 millimag; median: 0.7 millimag; best: 0.3 millimag) is comparable to very good ground-based astronomical sites.

In the 2010 and 2011 data, 93 lightcurves displaying transit-like structures were detected by the BLS algorithm, among which 8 are promising exoplanet candidates. Followup observations are in progress to ascertain their status.

\section{Acknowledgements}

The ASTEP project has been funded by the French agencies ANR (Agence National pour la Recherche), INSU (Institut National des Sciences de l'Univers), PPF-OPERA (Programme Pluri-Formation "Objectif Planètes Extrasolaires et Recherche en Antarctique") and PNP (Programme National de Planétologie). The authors are grateful to the French Polar Institute Paul-Emile Victor (IPEV) for their valuable logistic assistance.

\section{References}

Agabi K. et al. 2006, PASP, 118, 344

Alard C. \& Lupton R. H. 1998, ApJ, 503, 325

Andersen M. I., Freyhammer L., \& Storm J. 1995, "Gain calibration of array detectors by shifted and rotated exposures", ESO Conference and Workshop Proceedings, Proceedings of an ESO/ST-ECF workshop on calibrating and understanding HST and ESO instruments, 25-28 April 1995 P. Benvenuti Ed., p. 87

Aristidi E. et al. 2009, A\&SA, 499, 955

Baglin A. et al. 2006, 36th COSPAR Scientic Assembly, 36, 3749

Bakos G. A. et al. 2007, ApJ, 656, 552

Bellini A. et al. 2009, A\&BA, 493, 959

Borucki W. J. et al., 2010, Science, 327(5968), 977

Charbonneau, D., Brown T., Latham D., \& Mayor M. 2000, ApJ, 529, L45

Christian D. J. et al. 2006, Astron. Nachr., 327, 800

Crouzet, N., et al. 2010, A\&A, 511, A36

Crouzet N. et al. 2012, "An analysis of 4 years of data from ASTEP South", These proceedings. Charbonneau, D., Brown T., Latham D., \& Mayor M. 2000, ApJ, 529, L45

Hebb, L. et al. 2010, ApJ, 708, 224

Henry, G., Marcy, G., Butler, R. P., \& Vogt, S. S. 1999, IAU Circ. 7307

Kovacs G., Zucker S., \& Mazeh T. 2002, A\&A, 391, 369

Mandel K. \& Agol E., 2002, ApJ, 580, 171

Miller J. P., Pennypacker C. R., \& White G. L. 2008, PASP, 120, 449

Pont F., Zucker S., \& Queloz D. 2006, MNRAS, 373, 231

Ziad A. et al. 2008, A\&SA, 491, 917 